\title{
Uncertainties in complex dynamic environments
}

\author{
HASMIK ATOYAN \\ École Polytechnique de Montréal \\ JEAN-MARC ROBERT \\ École Polytechnique de Montréal \\ JEAN-RÉMI DUQUET \\ Lockheed Martin Canada
}

\begin{abstract}
Résumé : L'utilisation de systèmes d'aide à la décision (SAD) dans les environnements dynamiques complexes amène presque inévitablement l'opérateur humain à devoir faire face à plusieurs types d'informations incertaines. Il est alors essentiel pour les concepteurs de systèmes humain-machine : (i) de bien comprendre les différents types d'incertitudes qui peuvent exister dans les systèmes humain-machine qui font partie des environnements complexes, (ii) de connaître leurs impacts sur la confiance de l'opérateur dans les systèmes et sur son processus de décision, et (iii) d'avoir des lignes directrices pour savoir comment présenter des informations incertaines dans les interfaces utilisateurs. Il est aussi essentiel pour eux d'avoir une vue d'ensemble des étapes, des niveaux et des types d'automatisation de systèmes, et de connaître leurs impacts possibles sur la création de différents types d'incertitudes. Cet article traite de ces sujets et vise à aider les chercheurs et les praticiens à contrôler les incertitudes dans les environnements complexes.
\end{abstract}

Mots clés : Incertitudes, stratégies de raisonnement, lignes directrices, présentation d'informations incertaines.

\begin{abstract}
The utilization of Decision Support Systems (DSS) in complex dynamic environments leads the human operator almost inevitably to having to face several types of uncertainties. Thus it is essential for system designers to clearly understand the different types of uncertainties that could exist in human-machine systems of complex environments, to know their impacts on the operator's trust in the systems and decision-making process, and to have guidelines on how to present uncertain information on user interfaces. It is also essential for them to have an overview of the different stages, levels, and types of system automation, and to know their possible impacts on the creation of different types of uncertainties. This paper investigates these topics and aim at helping researchers and practitioners to deal with uncertainties in complex environments.
\end{abstract}

Key words: Uncertainties, reasoning strategies, guidelines, uncertain information presentation.

Les articles de JIPS sont publiés sous licence Creative Commons Paternité 2.0 Générique. 


\section{INTRODUCTION}

In the past, sometimes human operators could not perform their tasks effectively because of a lack of information. Today the problem is often the opposite: they are overwhelmed with information. The development of new technologies and their increasing use allow one receiving a vast amount of dynamic information. The human operator needs to monitor and assess this information, understand the current situation, infer possible future implications, and finally make decisions. For example, an air defence operator needs to monitor and analyze the information received from radars, and decide whether to engage a potentially hostile aircraft; or a risk analyst of financial market needs to evaluate various financial data and decide whether to buy or sell blocks of stocks. The situation is similar in medical diagnostics where the quantity of information is more that any single physician can absorb.

Decision Support Systems (DSS) can help human operators to handle the information overload, and support them in situation assessment and decision-making tasks. DSS help to organize the data in a meaningful way, analyze and correlate the information and make decisions more efficiently. Nevertheless, the deployment of automated systems does not necessarily simplify the operator's task and guarantee a better performance. It can have both positive and negative effects on human performance. It can lead to skills degradation, reduced situation awareness, and complacency (or overtrust) in the system. Many of these problems and of the accidents that can occur are discussed by [Sheridan and Parasuraman 2006] in their review of human-automation interaction.

There is another challenge that the DSS can pose to human operators. The automation can expose and even create several types of uncertainties, especially in dynamic complex environments. It is evident that people cope with uncertainties in data on a daily basis. They understand that the information upon which decisions are made could be incomplete, imprecise, and the decisions could be based on guesses and assumptions. However, in situations where the information is used to make high-risk decisions, the comprehensive understanding of data uncertainties and the quality of their presentation to the operator are vital.

In their discussions on visualization of uncertainties [MacEachren et al. 2005] stated that little had been done to understand the components of uncertainties and their relationships to domains, users and information needs, to assess the impact of visual depiction of uncertainties on decision-making process and decision outcomes, or to address the challenge of depicting multiple forms of uncertainties in the same display. They emphasized that "comprehensive guidelines for representing uncertainty do not yet exist" and that more research was needed to address all these questions. Riveiro [2007] emphasized that "most of the developed techniques to present uncertainties do not include a perceptual and cognitive analysis or user evaluation that validates their usefulness."

In this paper we identify and classify the different types of uncertainties that could be present in complex environments, we analyze them from a human factors perspective, and we examine their impacts on the operator's trust in the systems and decision-making process. Since uncertainties are often related to automation, we also present an overview of the different stages, levels and types of system automation and their possible impact on the creation of different types of uncertainties. Finally, we propose system design guidelines for helping human operators to cope with uncertainties. The goal of the paper is to help researchers and practitioners to deal with uncertainties in complex environments.

Journal d'Interaction Personne-Système, Vol. 2, Num. 1, Art.5, Janvier 2011 


\section{DIFFERENT TYPES OF UNCERTAINTIES}

In order to understand how to design systems that could help the operators to cope with uncertainties we need to identify the types and sources of uncertainties.

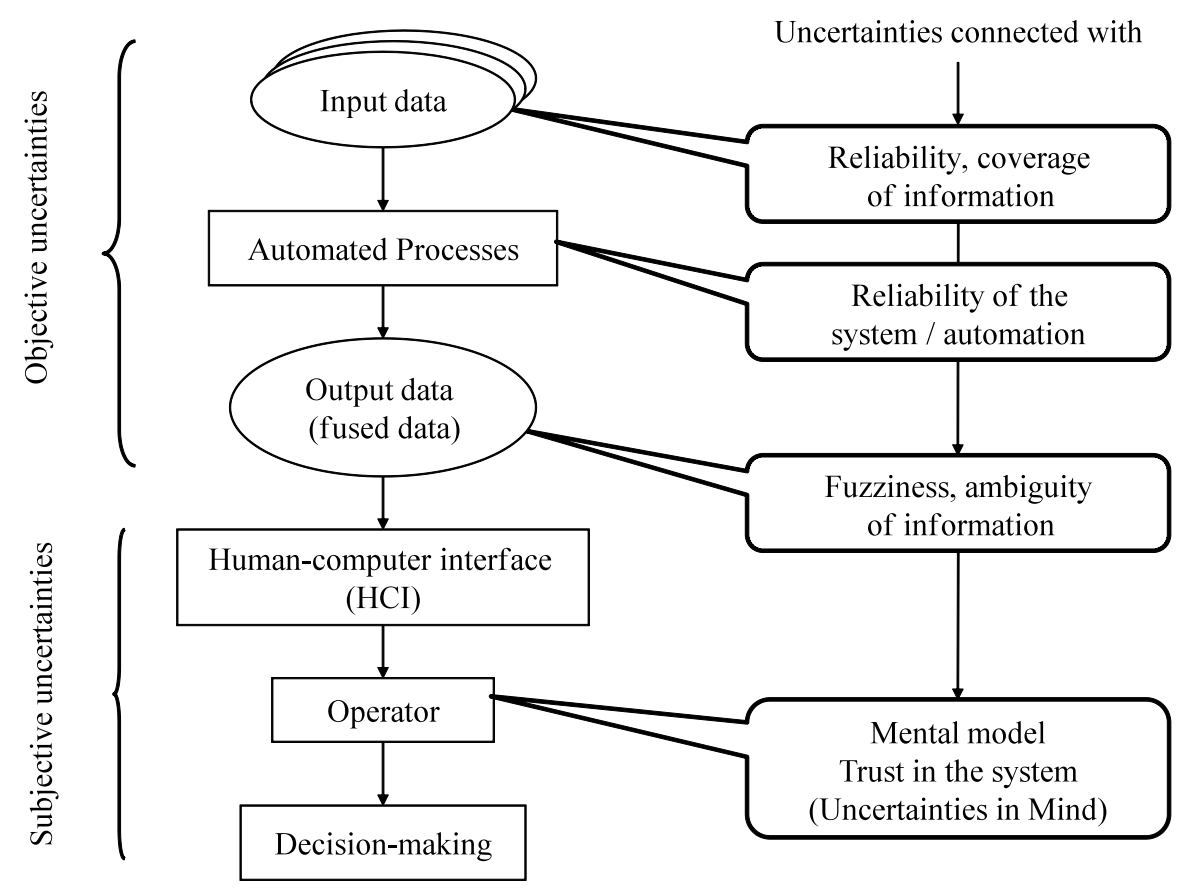

Figure 1. Different types of uncertainties

There are different types of uncertainties that could surface during the decision-making process. In Figure 1 we show the two types of uncertainties that can be distinguished in complex dynamic systems, namely objective and subjective uncertainties [Atoyan et al. 2009]. The objective uncertainties include those that are connected with the system itself, namely:

- Uncertainties connected with the input data, i.e. the information that is received from different sources by the system. For example, in military domain it could be information received from the radars, from human intelligence sources, from different coalition forces, etc. All this information will have different levels of precision, reliability, and credibility.

- Uncertainties connected with automated processes. There will always be a set of conditions (e.g., system malfunctions, environmental conditions, etc.) under which the automation can yield incorrect results [Wickens and $\mathrm{Xu}, 2002]$. Hence, the automated systems have different levels of reliability. 
- Uncertainties connected with the fused output data. This output is the correlation of the received information which is a statistical average. The fused output could be the likelihood of future events or ambiguous information (for example, $30 \%$ chance that there is a target and $70 \%$ that there is not).

As it is shown in Figure 1, another type of uncertainty that shows up in decision-making tasks is the subjective uncertainty, i.e. uncertainty in the mind of the operator. There are always differences in how people perceive and process the information, which in turn affect the operator's decisions and actions. These differences are due to such subjective elements as the operator's expectations, mental model and trust.

In the following section we present a detailed analysis of these different types of uncertainties.

\subsection{Uncertainties Associated to Data Acquisition}

The uncertainties in data acquisition are examined and discussed by different authors [Riveiro 2007], [Sager et al. 2007], [Griethe and Schumann 2005], [Thomson et al. 2005]. They could be associated to:

- The lack of precision of data acquisition sources and methods;

- Information processing and transformation of information (e.g., errors or reduction of precision during information processing);

- Inconsistency between the information from different sources;

- Temporal delay between occurrence and information acquisition, etc.

Humans expect that automated systems should perform in a near perfect way [Dzindolet et al. 2003]. And if they are not aware of the system possible errors (because of information imprecision or errors in system during information processing) it could trigger a rapid decline of trust in the system [Dzindolet et al. 2003], [Madhavan and Wiegmann 2004]. This may hinder the operator in making the right decision.

\subsection{Uncertainties Associated to Data Fusion}

In order to handle the uncertainties and the missing or conflicting data, different probabilistic methods are being applied during the information fusion process. The data from multiple sources are combined (integrated) by utilizing different reasoning algorithms and presented to human operators. The data integration process can contain several types of uncertainties.

[Klir and Wierman 1999] divide uncertainties associated with data integration into two categories: fuzziness (implies elements for which it is not possible to decide if they belong to certain category or subset) and ambiguity (lack of agreement and difficulty to discriminate between the elements of a category). These sources of uncertainty can be reduced by the fusion process (e.g. reduced fuzziness), but they remain part of the fused information products and can even become more prevalent in the end system. For example, in military systems the end results may include estimated list of identity options (e.g. $40 \%$ hostile target vs. $60 \%$ friendly target) with associated likelihood or evidence of conflicting sensor data, etc. All these situations represent new types of uncertainties that had not been 
necessarily present in the initial data but that need to be brought to the attention of the decision maker.

If the operator is used to fuse the data mentally then there would be new challenges for $\mathrm{him} / \mathrm{her}$ if the reasoning of the system is different from his/her usual reasoning process. The reasoning of the automated system can become a black box for the operator and create another type of uncertainty for him/her.

\subsection{Uncertainties associated to system reliability}

Automation can vary in level, starting from lower level of automation where it presents only the relevant data to fully automation where the operator is not involved in decision processes. [Sheridan and Verplank 1978] have proposed the following scale of levels of automation (LOA):

1. Human does it all;

2. Computer offers alternatives;

3. Computer narrows alternatives down to a few;

4. Computer suggests a recommended alternative;

5. Computer executes alternative if human approves;

6. Computer executes alternative, human can veto;

7. Computer executes alternative and informs human;

8. Computer executes selected alternative and informs human only if asked;

9. Computer executes selected alternative and informs human only decides to;

10. Computer acts entirely autonomously.

The automation can differ in type and complexity, from simply organizing the information sources to integrating them in some summary, to giving advices and recommended choices, or even to providing course of action. Therefore, [Parasuraman et al. 2000] have proposed an extension of the LOA concept. They proposed four information processing stages where each stage can have its own LOA. The four stages (also called "types" by many authors) are:

1. Information acquisition: collection and filtering of information (processing, preprocessing of data);

2. Information analysis: integration of information in a form of inference or diagnosis (integration, diagnosis, and inference, occurring prior to the point of decision);

3. Decision and action selection: selection among decision alternatives;

4. Action implementation: execution of chosen action.

If we compare these stages with data fusion process, then the first stage of automation is related to input data and the data fusion output could not only be information analysis but also decision and action selection or actions implementation.

Higher are the level and stage of automation higher are the operators' concerns about the reliability of the system. It is possible for the operator to observe the process of information acquisition, i.e. stage 1, even if he/she is not relying on it [Yeh and Wickens 2001]. At this stage the raw data are still available, so here the operators can balance their attention between the raw data and automation [Wickens et al. 2000]. In contrast, it is not possible for the operators to observe action implementation, i.e. the stage 4 , unless they are relying on it [Lee and Moray 1992]. Uncertainties in reliability of the system might increase the 
probability that the operator will disagree with the system results, resulting in its distrust and disuse of the system [Atoyan 2006].

When the operators are aware of the automation faults or imperfections, they can more carefully calibrate their allocation of attention (automation stages 1 and 2) or be prepared for incorrect system actions (automation stages 3 and 4) due to imperfect automation [Wickens and $\mathrm{Xu} 2002]$. The importance of feedback to an operator about automation errors was also shown in studies conducted by [Simpson 1992] in the domain of naval Command and Control systems. The same findings have been shown by [Dzindolet et al. 2003]. They found that providing operators with information about the conditions in which an automated aid is likely to make errors leads to an improved task performance.

\subsubsection{Adaptive and Adaptable Automation}

An important issue with regard to human-automation interaction is the role of automation during system operations. To improve the human-automation performance the use of adaptive and adaptable automation has been proposed by different authors [Miller and Parasuraman 2007], [Miller et al. 2005], [Kaber and Endsley 2004], [Moray et al. 2000] and [Opperman 1994]. In adaptive or adaptable automation the types and levels of automation are not fixed, but dynamic. They may change during the system operations. However, there is a difference between adaptive and adaptable automation.

In adaptive automation the decisions to invoke automation or to return automated task control back to human is defined by the system. For example when high human workload is detected or when a human is not responding appropriately, automation can switch to a higher level and become less dependent on the operator.

In contrast, in adaptable systems the operator retains the authority to invoke or change the automation. Here the operator is in charge and decides how much automation to use, and can change the LOA when necessary. [Miller and Parasuraman 2007] argue that the adaptable automation can lead to the same benefits as adaptive automation while avoiding many of its pitfalls. According to various authors the adaptive automation raises the issue of human acceptance of such a system. Many users may be unwilling to accept the system driven adaptation. These adaptive changes performed by the system may increase the system's unpredictability to the user [Billings and Woods 1994]. Unpredictability of the automation behaviour will add more uncertainties for the operator with regard to the system behaviour.

By keeping the operator active in charge of how much and what kind of automation to use and when to use it, we keep the operator "in the loop" and develop appropriate level of trust in the system [Atoyan et al. 2006], [Atoyan 2006], [Dzindolet et al. 2003], which means trust that is neither too high nor too low. Both overtrust and undertrust can compromise the safety and profitability of the system [Atoyan et al. 2006], [Lee and See 2004], [Parasuraman and Riley 1997].

\subsection{Uncertainties Associated to State of Mind}

Uncertainties in decision-making process could be formed by the mental models of individuals. Mental models are psychological devices used to describe how individuals structure and explain the world around them. Mental model is a key factor in determining how individuals will behave in any situation. Human mental model is qualitatively different 
from computer based models. Human mental model contains not only information but also other psychological variables that humans will use to structure and interpret the world around them. This includes subjective elements such as belief and personal bias, group influence, cultural expectations. In fact, these subjective elements mostly interfere during abnormal situations [Merritt 2000].

Decision-makers have certain mental models about the system with which they interact. These mental models comprise the operators' understanding of system objective(s), its functionalities and the logic of system decision making. If the logic of the system reasoning is not consistent with the mental model of the operator on how the system makes decision than several uncertainties will be created in the mind of the operator. This will impact operator's trust in the system. Operator might consider the system untrustworthy even if the system reliability will be high [Dzindolet et al. 2003]. This will impact operator's performance, and could also cause disuse of the system.

Nevertheless, even if the system reasoning will be consistent with the mental model of the operator, human decision-maker will always assess, and thus internalize a subjective notion of the uncertainty represented by the data fusion output. And as with assessments of subjective probabilities, this subjective factor may be impacted by the manner in which the information is presented [Bisantz et al. 2002]. Therefore, it is important to analyze the user expectations regarding to information presentation.

To design DSS that are well adapted to users' mental model, we need to understand the human reasoning and expectations with regard to the system and information presentation. In the next two sections we present discussions on human and system reasoning and presentation of uncertain information.

\section{REASONING STRATEGIES USED BY HUMAN DECISION MAKERS AND AUTOMATED SYSTEMS}

To get more insight on the consistency between human and system reasoning, first we need to define and analyse various reasoning strategies used by humans and by automated systems, and then to establish commonalities and divergences between them.

\subsection{Classical Decision-Making Strategies}

Human decision makers have a toolbox of different heuristics. When facing a problem, an individual must choose an appropriate reasoning strategy for a given situation. These strategies can be classified as compensatory and non compensatory.

Compensatory strategy is the processing in place when a good value of one attribute can compensate a poor value of another (e.g. the car has a high price but low gas consumption). In compensatory strategy, multiple attributes of a particular option are considered before another alternative is examined. Compensatory strategy of human reasoning is close to normative reasoning used in decision aid systems.

During non-compensatory reasoning the values of several alternatives on a single attribute are processed before the information of another option is examined. In case of noncompensatory reasoning, people do not make trade-offs between the attributes and often do not assess all the attributes but only the most important ones, and choose the option where the attribute has the highest value. 
Examples of non-compensatory strategies are LEX (Lexicographic Strategy), SAT (Satisficing Strategy), EBA (Elimination by Aspects), MCD (Majority of Confirming Dimensions), etc. Examples of compensatory strategies are WADD (Weighted Adding Strategy) and EQW (Equal Weight Strategy). When facing what strategy to implement, the decision maker evaluates the present tools, the benefits and costs, the available time, and selects the one that is best fitted to solve the problem. People also can use a combination of strategies [Payne and Bettman 2001].

\section{Results associated to compensatory and non-compensatory strategy selection}

Several authors have studied strategy selection depending on different environmental and task variables. Some of these results are presented in Table 1.

The utilization of the compensatory or non-compensatory strategy can depend on the manner in which the graphical display is organized. People can use a less important cue simply because it is more salient in the display [Jarvenpaa 1989]. Hence, the strategy applied by the operator is not always the best, it can be adapted to the information presentation.

We can conclude that the decision strategies utilized by decision-makers depend on such factors as the task, context demands, needed accuracy, personal differences, time constraints, level of risk involved, experience, knowledge of different strategies, and presentation of information. Any changes in these factors can cause changes in the strategy. However, it is not guaranteed that the strategy utilized by the operator is optimal, since the operator could have adapted himself/herself to the one provided by the system, or she/he could have limited experience with alternative strategies

Table 1. Factors affecting the choice of strategy.

\begin{tabular}{|l|l|}
\hline Strategy & Factors affecting the choice of strategy \\
\hline $\begin{array}{l}\text { Compensatory } \\
\text { (requires high level } \\
\text { cognitive resources) }\end{array}$ & $\begin{array}{l}\text { When multiple attributes are presented simultaneously [Lurie } \\
\text { and Mason 2007]. }\end{array}$ \\
& $\begin{array}{l}\text { When facing a decision problem involving two or three } \\
\text { alternatives [Lurie and Mason 2007]. }\end{array}$ \\
& $\begin{array}{l}\text { Linguistic presentation of attribute values leads to lesser } \\
\text { compensatory processing than the numerical presentation of } \\
\text { values [Stone and Schkade 1991]. } \\
\text { The interactive visualization tools (enabling the user to } \\
\text { restructure the information environment; i.e. when the } \\
\text { computer allows users to interact with plots, changing } \\
\text { parameters, querying, zooming and linking plots) aim at } \\
\text { lowering the cognitive load and can lead to more extensive } \\
\text { compensatory processing and more accurate decisions [Eick } \\
\text { and Wills 1995]. } \\
\text { The WADD strategy is robust in terms of accuracy across a }\end{array}$ \\
\hline
\end{tabular}

Journal d'Interaction Personne-Système, Vol. 2, Num. 1, Art.5, Janvier 2011 


\begin{tabular}{|l|l|}
\hline Strategy & Factors affecting the choice of strategy \\
& $\begin{array}{l}\text { variety of decision environments. This strategy is especially } \\
\text { efficient when there are negative correlations between } \\
\text { attributes [Payne and Bettman 2001]. } \\
\text { In the case of conflict between the attributes people use more } \\
\text { extensive processing strategies. They apply less selective and } \\
\text { more alternative-based processing and their decision accuracy } \\
\text { is higher [Lurie and Mason 2007]. }\end{array}$ \\
\hline $\begin{array}{l}\text { Non-compensatory } \\
\text { (requires moderate } \\
\text { level cognitive } \\
\text { resources) }\end{array}$ & $\begin{array}{l}\text { When only a few attributes are presented simultaneously } \\
\text { [Lurie and Mason 2007]. }\end{array}$ \\
$\begin{array}{l}\text { When facing complex multi-alternative decision tasks, people } \\
\text { tend to use non-compensatory strategies, such as SAT and } \\
\text { EBA [Johnson et al. 1989]. } \\
\text { During time-constraint situations, the compensatory strategy } \\
\text { is less accurate than other non-compensatory strategies } \\
\text { because the processing is shortened. The EBA strategy is } \\
\text { remarkably robust to variations in time pressure [Payne and } \\
\text { Bettman 2001]. }\end{array}$ \\
\hline
\end{tabular}

\subsection{Coping with Uncertainties in Naturalistic Settings}

In real world context there are deviations from classical mechanistic models of problem solving [Klein 1998]. Formal analytical models can yield optimal solutions with great precision and rigor, however, they can also be inefficient owing to the cognitive effort which they require, their poor compatibility with the non-analytic cognitive processes which decision makers typically use [Hammond 1993]. People tend to deviate systematically from the rational choice model even when presented with relatively simple tasks [Kahneman et al. 1982].

Naturalistic Decision-Making (NDM) is an attempt to understand how people make decisions in real-world contexts that are meaningful and familiar to them. [Orasanu and Connolly 1993] list eight factors which they claim characterize naturalistic settings. These factors are:

- Ill-structured problems;

- Uncertain, dynamic environments;

- Shifting, ill-defined, or competing goals;

- Multiple event feedback loop;

- Time constraints;

- High stakes;

- Multiple players;

- Organizational goals and norms.

NDM models do not attempt to predict which option will be implemented, but describe the cognitive processes of proficient decision makers. Studies of the decision-making process of experts have yielded that typically they make decisions by various forms of 
matching (i.e. "Perform action A because it is appropriate for situation S") and not by concurrent choice (i.e. "Perform action A because it has superior outcomes to its alternatives") [Lipshitz 1994].

[Lipshitz and Strauss 1997] examined 102 cases where decision makers were facing uncertainties such as inadequate understanding of the situation, incomplete information, and presence of conflicting alternatives. They call this RAWFS heuristic (Reduction, Assumption based reasoning, Weighing pros and cons, Forestalling, and Suppression). To cope with these uncertainties, decision makers used five strategies:

- $\quad$ Reduction (trying to reduce uncertainty; e.g. collecting additional information);

- Assumption-based reasoning (relying on knowledge and imagination to fill in gaps, or make sense of factual information);

- Weighing pros and cons of at least two competing alternatives (comparing the merits of competing alternatives);

- Forestalling (preparing a course of action to counter potential negative contingencies, (e.g. building reserves or preparing a worst-case option);

- Suppressing uncertainty (e.g. by ignoring it or by "taking a risk"). This option is the last resort.

Most NDM models assume some level of expertise in the field, not necessarily that of an expert, but definitely not that of a novice. Analyzing the experimental results of different studies related to differences between expert and novice decision makers, [Lipshitz and Cohen 2005] conclude that:

- Experts are more likely than novices to build stories, to step back and evaluate the story, and if necessary to build an alternative story;

- Novices are more likely than experts to use checklists;

- Experts are more likely than novices to take certain specific types of information into account: this includes big picture components that affect the intention of actors;

- Experts are more likely than novices to consider the amount or time available for decision making and to adjust the time taken accordingly.

While working in complex environments, the human decision makers can always face situations where time constraints, high stakes, multiple players, ill-structured problems, and uncertain dynamic situations are present. It means that the operators, especially the experienced ones, will usually apply informal naturalistic approach. During regular situations when the environmental circumstances will allow utilization of analytical formal reasoning, the human operator will usually apply formal mechanistic reasoning strategy. This strategy will be used by novice operators in most situations and by experienced operators in new unanticipated situations.

Another important issue to be noted is that the operator should always be aware of potential uncertainties. NDM approach always mentions the importance of right situation awareness, that the decision maker should already have assimilated all the relevant information when the decision has to be made rather than being given the relevant information only at that time. This suggests that uncertainty should be presented continuously and not only when the decision has to be made. 


\subsection{Reasoning Algorithms}

The comprehensive understanding of the algorithms used by the automation may support the operator not only to identify how the system arrives to decisions, but also why the automation might make an error [Atoyan et al. 2006], [Dzindolet et al. 2003]. It is understandable that in many cases the operator's background knowledge in mathematics could be insufficient to understand the probability models and theories utilized in the algorithms. Hence, the system should provide the operator with the general logic and comprehensive instructions of the inference that is being utilized. Furthermore, taking into consideration the importance of the mental model in decision-making tasks, it would be preferable to select an algorithm with minimum mismatches with the human reasoning in the given context. The lesser the mismatch between the automation and human reasoning, the lower the operator's cognitive load will be.

DSS developers use different algorithms to develop automated systems. For example, the probabilistic methods largely used in DSS applications are Bayesian inference, Depmster Shafer (D-S) inference, and fuzzy logic. Most Artificial Intelligence applications use the model of neural network (NN). Each method has its own characteristics, advantages and disadvantages. We describe some of them.

- The computational model of NN is very efficient in classification, novelty detection, and data processing (as filtering, clustering) tasks. However, its disadvantage is that the reasoning process of the system, the relation between the input and output variables remains a blackbox for the operator.

- The method of fuzzy logic is a new way of expressing probability. It is an organized and mathematical method of handling inherently imprecise concepts. The concept of "coldness" cannot be expressed in an equation. Fuzzy logic is the same as "imprecise logic".

- Bayesian networks and D-S inference both allow representation of knowledge in uncertain domains. Bayesian networks normally need a complete knowledge of real world to build a correct model. They require prior probabilities for every single event (i.e. rigorously defined numbers that reflect how often an event will happen if an experiment is performed a large number of times). Bayesian method is restricted to the classical notion of probabilities, it does not use a specific state called "unknown", and it considers that something (some event) either is or is not true.

- For D-S no prior probability is required. However, it requires masses (the initial knowledge of the system) to be assigned to the various alternatives. D-S also allows the computation of the higher and lower bounds of beliefs (belief and plausibility). It explicitly allows for an undecided state of our knowledge.

[Koks and Shalla 2005] compare Bayesian and D-S inferences and state that "Unlike Bayes theory, Dempster-Shafer theory explicitly allows for an undecided state of our knowledge. It can of course sometimes be far safer to be undecided about what a target is than to decide wrongly and act accordingly with what might be disastrous consequences".

Choosing between different algorithms is not trivial, there could be several tradeoffs. In order to decrease the mismatch between the reasoning of automation and the reasoning of the human, one has to analyze the environmental and task constraints, the user characteristics and the reasoning strategies, and select the reasoning algorithm from a human factors perspective, i.e. that will best match to that particular profile. 


\section{INFORMATION PRESENTATION AND DECISION-MAKING}

Information presentation has a strong impact on the decision-making processes [Lurie and Mason 2007], [Gilovich et al. 2002], [Bisantz et al. 2002], [Kahnman and Tversky 2000], [Gigerenzer, 1994]. For instance, more vivid visual information is likely to be acquired and processed before less vivid information [Jarvenpaa 1990], [Mandel and Johnson 2002]. Humans can process more information when it is presented graphically rather than in textual form [Tegarden 1999]. It is easier to detect interactions between different features in graphical displays than in verbal descriptions [Holbrook and Moore 1981]. Analysing different visualization techniques, Lurie and Mason [2007] assert that:

- When visualization includes both text-based and graphic information, the latter receives greater weight;

- Presentation of information in both graphical and tabular formats leads to higher performance in terms of decision speed and accuracy compared with the case of either graphic or tabular formats alone;

- Vivid information is more likely to be overweighted by novice than by expert users, and the superiority in the priority for graphical versus text-based representation is likely to depend on the decision maker's expertise.

Ordering the cues from the most to the least important helps to create display conformity with the user expectations and facilitates building a coherent story that explains the evidence, and thus facilitates the decision making [Adelman et al. 1996], [Liebhaber and Feher 2000], [Perrin et al. 2001].

[Payne et al. 1999] found that even though a great deal of information may be available, human processing limitations require selectivity. The overload with too much information may trigger a utilization of simplifying heuristics, where the user can fail to focus the attention on important information. Hence, it is essential to define the information that is most critical for the user to achieve the objectives of the task. [Eick and Wills 1995] found that an interactive display enabling the user to restructure the information environment may support information acquisition that more closely reflects the decision maker's pre-existing preferences or knowledge structures. All these should reduce the cognitive load and improve the decision quality.

\subsection{Visualization of Uncertainties}

As we mentioned above, the operator should always be aware of existing uncertainties. Otherwise, he/she has to check the validity of the data all the time. [Andre and Cutler 1998] found that if uncertainties are not presented, the operator tends to adopt risky behavior. They assert that operators have a tendency to ignore hidden/implicit decision criteria when they are not visually presented.

To date, there has been limited research on how to present uncertainties to support the decision-making process, particularly in object identification tasks. Uncertainties can be presented by different modalities; [Basapur et al. 2003] investigated the impact of visual (color coding), auditory (tones) and haptic (vibration) modalities of presentation of uncertain information on performance, trust and workload. The experimental results revealed that the overall workload was the same for all modalities. However, the haptic modality resulted in higher physical workload, and the auditory created higher level of frustration. Measures of 
level of trust were similar for all modalities in the beginning, but did tend to decrease over time, indicating that participants began to more clearly recognize the uncertain nature of the information they were receiving.

\subsubsection{Graphical Presentation of Uncertainties}

Graphical representations seem to grasp attention very quickly. Hence, in uncertain situations where the focus or key elements of the decision may change quickly, the utilization of graphical representation could be more efficient to direct the intentional processes than other means. One candidate graphical representation is the use of blurred or degraded graphical images. [MacEachren 1992] suggested using blurred lines to indicate uncertain boundaries and blurred images to indicate uncertainty in a type of land cover. Such a technique may be useful in conveying uncertainty because the manipulation of the image (e.g. through blurring or fuzziness) corresponds in a natural way to the level of uncertainty regarding the data. In his book [Norman 1988] discusses the advantage of natural mappings in the design of displays. Findings of [Lind et al. 1995] show similar results. They found that pilots' estimates of weather hazards increased as the graphical distortion increased. [Bisantz et al. 2002] propose that presenting uncertainty on information visually through blurred icons could provide the operator with a quick understanding of the level of threat and uncertainty relative to that threat. Then, the system can provide more information upon the demand of the decision maker. So far, there has been no empirical investigation on the usefulness of this technique. Figure 2 presents an example of blurred icons.

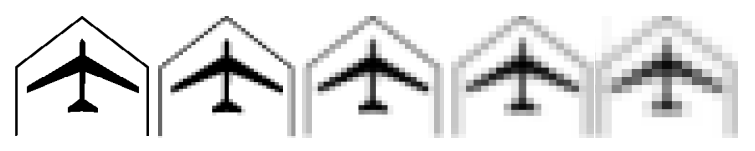

Figure 2. Example of the blurred icon approach

The investigations of the U.S. Navy air threat assessment [Liebhaber and Feher 2000] revealed the navy officers' preferences for graphical presentation, namely for graphical bars (Figure 3) when the provided information includes uncertainties. The officers felt that threat assessment is a fuzzy concept, therefore it should not be indicated in numbers. Numbers, in their opinion, implied false sense of accuracy and the graphical format can support in avoiding that false sense of precision.

Linguistic representation of uncertainties consists in using words such as "likely" "probable" "unlikely". Such representation can be described as vague representation of uncertainty, compared to precise representation such as numeric probability values [Wallsten 1990], [Wallsten and Budescu 1995]. These authors showed that there are individual differences in the manner in which people both interpret and generate linguistic expressions of probability values. [Dila and Stone 1997] found that the decision maker's agreement on cue weights and judgment consensus are higher with cue information stated in words rather than in numbers, since appropriately chosen words can more directly convey the risk implications of information cues. They also found that linguistic presentation of 
information increased the time required to acquire cue information relative to numeric representation.

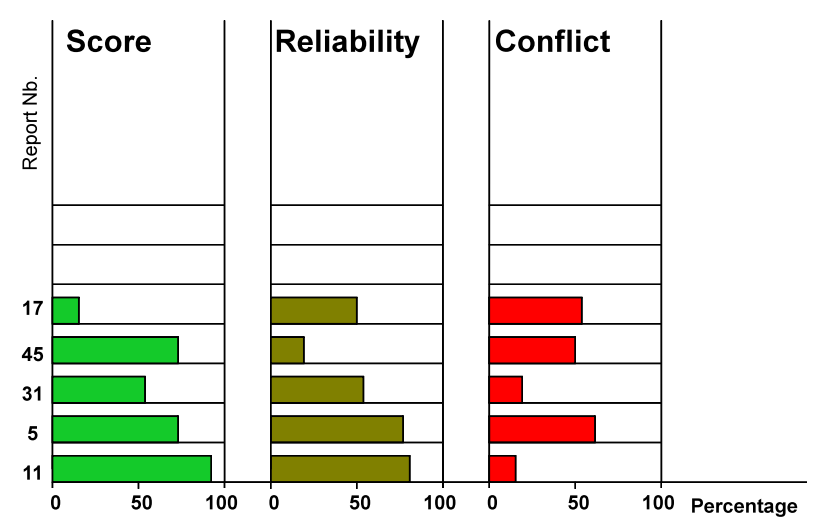

Figure 3. Examples of graphical bars used for target identification in intelligence analysis (score of the information correlation result for the target; reliability of the information; correlation conflict)

Some researchers have found that the performance on frequentist versions of problems was superior to non-frequentist versions, i.e. presenting uncertain information in a frequency format (e.g., 1 out of 10) rather than in a probability format (e.g., .1) improves the participants' performance [Cosmides and Tooby 1996], [Gigerenzer and Hoffrage 1995]. They connect this effect with the fact that frequentist reasoning mimics the type of probability information people can gather naturally. On the other hand, the experimental results of [Bisantz and Kirlik 1998] on identification of aircraft identity revealed better performance for probability format, especially if the operator has task-related experience. This is inconsistent with the results of previous authors. [Bisantz and Kirlik 1998] assert that it could be connected with the differences of experimental conditions.

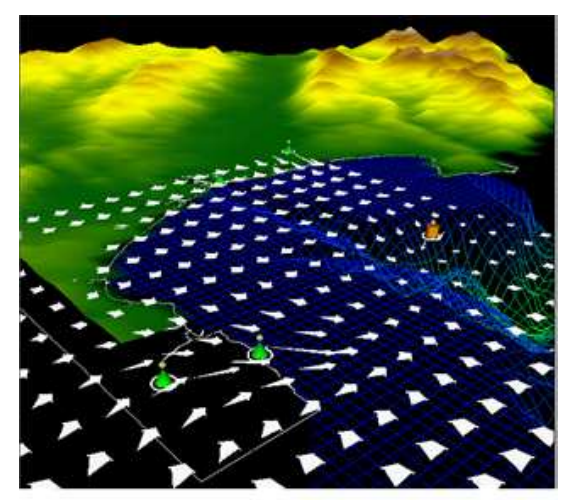

Figure 4. Examples of glyphs of winds and ocean currents (Reproduced with permission) 
So far, the research on graphical presentation of uncertainty has been mainly conducted in the domain of geographical information systems. [Pang et al. 1997] suggested the use of glyphs (graphical forms such as arrows or vertical lines) to display the uncertain data (Figure 4). Providing information uncertainty directly in the glyph can allow other graphical dimensions (color, texture) to be utilized for other purposes.

Table 2 shows examples of presentation of uncertainties in four different formats, in the domain of probabilistic reasoning. The numerical, frequentist, and linguistic formats can be used in visual and auditory presentations.

Table 2. Examples of presentation of uncertainties in four different formats

\begin{tabular}{|l|l|}
\hline \multicolumn{1}{|c|}{ Formats } & \multicolumn{1}{c|}{ Examples } \\
\hline Graphical & See Figure 2 \\
\hline Numerical & $0.1 ; .1 ; 1 / 10 ; 10 \% ; ; 1 \div 100$, etc. \\
\hline Frequentist & 1 out of 100 \\
\hline Linguistic & $\begin{array}{l}\text { Likely, Possible, Unlikely } \\
\text { Low, Medium, High; etc. }\end{array}$ \\
\hline
\end{tabular}

In their discussions relative to the state of art of visualization of uncertainties [MacEachren et al. 2005] assert that little has been done to understand, assess the impact of visual depiction of uncertainties on decision-making process, decision outcomes or to address the challenge of depicting multiple forms of uncertainties in the same display. They emphasize that "comprehensive guidelines for representing uncertainty do not yet exist" and more research is needed to address all these questions.

\section{UNCERTAINTIES AND DECISION-MAKING}

The discussion above shows that the uncertainties have different characteristics at different stages of automation. Figure 5 presents the relation between uncertainties and different stages of automation.

At the stage of information acquisition (input data) uncertainties are connected with:

- Quality of information, i.e. the precision, accuracy and reliability of information received by different sources (e.g., the radars have different levels of precision, the intelligent sources have different levels of reliability, etc.).

- Missing information, i.e. the information that is required for decision making but is not available (e.g., in military domain it can be the lack of information about the target platform; in medical domain it can be the missing information about the patients family history, etc.)

- Reliability of the system automation can vary in reliability, therefore the level of uncertainty with regard to the system will depend on the level of reliability of the system.

At the stages of information analysis, decision selection and action implementation uncertainties are connected with: 
- Fused output of the system, i.e. the correlated output of the received information, which is a statistical average.

- Operator's understanding of the system reasoning, i.e. understanding the logic of the system.

- Reliability of the system.

Finally, there are subjective uncertainties or uncertainties in the mind of the operator. They are connected with:

- Consistency between the presented information and the operator's expectations;

- Consistency of the system logic with the operator's reasoning;

- System reliability and the operator's mental models, expectations and trust in the system.

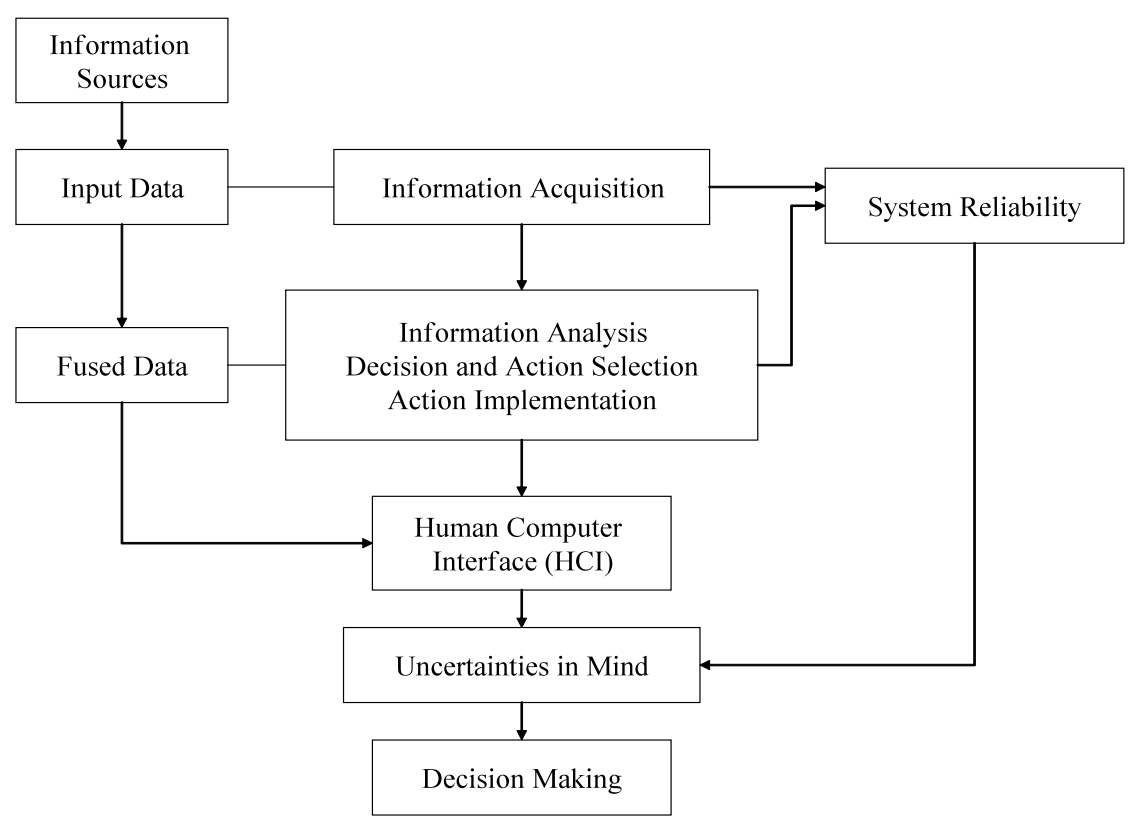

Figure 5. Uncertainties associated to complex systems

\section{HIGH LEVEL DESIGN GUIDELINES FOR COPING WITH UNCERTAINTIES}

Uncertainties in complex systems should be analyzed not only for deciding how to present them on human-computer interfaces but more importantly for designing fundamental aspects of the system such as the reasoning algorithm selection, the traceability of the input 
information, the information relative to the data precision, and the levels and stages of automation.

Based on a review of several theoretical, empirical and analytical studies which present different types of uncertainties in systems and the challenges that they can create for the operators, we propose several system design guidelines to help operators to cope with uncertainties. The guidelines are organized in four groups on issues related to input data, output data, automated processes, and human-computer interface.

Input data

1. Provide information on data precision, reliability and credibility, on possible errors connected with data processing or information transformation, and on the missing data.

This will reduce the uncertainties that the operators could face while monitoring the system. If the operators are aware of incompleteness, imprecision of the data, system errors, or that data are missing they can develop a control strategy to compensate for such errors. The knowledge of uncertainties in input data will also help to develop an appropriate level of trust towards the automated system.

2. Provide an access to raw data, their accuracy, precision and reliability at all stages and levels of automation.

This does not mean visualization of this information on the main display at all stages of automation. Nevertheless, it is important for the operator to know that if needed he/she can trace this information. The danger of unavailability of raw data is that the operators have no other choice but to either totally rely (misuse) on automation or ignore it (disuse).

Output data

3. Find means to show the operator the reasoning of the system, the logic of the fusion process in a way that will be understandable to him/her. For example, in target identification task this can be done by providing the operator the attributes that are being fused (speed, altitude, platform ... of the target). If the fusion uses also the environmental context then provide the information used (region, hostile or friendly forces around, etc.) for this level of the fusion.

This will help the operator to understand how the system arrives to decisions and will reduce the uncertainties in the mind of the operator associated to data fusion output. The automation will be more trusted if the operator understands the algorithms underlying the automation.

4. Identify the reasoning strategy/strategies utilized by the human operator in the decisionmaking process, and try to adapt the reasoning logic of the algorithm to the reasoning utilized by the human operator. Cognitive task analysis is an efficient method to reveal the reasoning of the operator. It can reveal the key information needed by the operators during their reasoning while performing the tasks. It can help to understand whether operators prefer to get a final answer "X\% hostile and Y\% neural" or answer with the option of unknown such as " $\mathrm{X} \%$ hostile, $\mathrm{Y} \%$ neural and $\mathrm{Z} \%$ unknown (see section 3.3).

The lesser the mismatch between the reasoning methods of the system and of the human decision maker, the lesser the resulting subjective uncertainties for the latter.

Automated processes 
5. Provide information about automation faults, imperfection, or the conditions in which an automated aid is likely to make errors.

This can help the operators to calibrate their allocation of attention or preparation for incorrect actions to automation imperfection. This will also help to reduce uncertainties in the operator's mind with regard to system reliability and help to develop appropriate level of trust in the system. Note that higher are the stages and levels of automation higher are the levels of uncertainties created by the system.

6. Adaptable automation can help to reduce uncertainties in the system.

This approach can help to implement automation at all stages and levels but at the same time help the operator be in charge of how much and what kind of automation to use. This will support the operator to avoid unpredictability of the system. When people are in control of automation, they can participate in the decision-making process and intervene when needed. This will reduce the uncertainties. It will also help to develop an appropriate level of trust in the system.

Human-computer interface

7. Present uncertainty continuously, not only when a decision has to be made.

8. Enable the user to restructure the information. For example, allow the user (especially the expert user) to re-organize the information of the display (e.g., provide a filter to display all information or only information needed for the context).

9. Support not only structured (mechanistic) models of decision-making but also the intuitive (naturalistic) judgment of human operators

10. Support the compensatory information processing strategy in case of small amount (e.g., two or three) of alternatives. Interactive visualization tools can lead to more compensatory processing and more accurate decisions. Different representations of each cue lead to more of a compensatory processing strategy in decision-making than the presentation of all cues in the same manner.

11. Support non-compensatory strategy in case of complex, multi-alternative decision tasks. Linguistic presentation of attribute values leads to less compensatory processing than the numerical presentation of values.

12. Define the most important cues utilized by human operators for the task, and order them by importance to the decision maker. Make sure that less important cues are not more salient.

13. Decision makers' agreement on cue weights and judgment consensus is higher for linguistic presentation than for numeric presentation. Appropriately chosen words can more directly convey the risk implications of information cues.

14. Since uncertainty is a fuzzy concept, its graphical or linguistic (e.g. low, medium, high) presentation can support the operator to avoid the false sense of precision. However, note that decision makers can both interpret and generate linguistic expressions of probability values differently because of individual differences between them. People base their interpretation of linguistic probabilities on their experience with the meaning of the linguistic expressions. 
15. Linguistic presentation of information can increase the time required to acquire cue information relative to numeric representation.

16. Graphical distortion (e.g., less resolution) to present uncertainties can improve performance. This technique corresponds in a natural way to convey the level of uncertainty regarding the data.

17. Presenting uncertain information in a frequency format rather than in a percentage format could improve the performance. However, performance on the probability estimation could be better in the latter format for users with task related experience.

18. Design systems and procedures that can minimize the effect of individual cultural variability with regard to uncertainty management. Analysis of the user profile (e.g., education background, work experience, work domain, etc.) will reveal the user's preferences and expectations.

19. Provide adequate training. It is not always possible to fit the presentation to the cognitive model of the decision maker. In these cases the operators need an appropriate training that will help them to change their mental model. Provide the operators the right training to understand system's goals, functionalities, reasoning procedures, possible shortcomings, anomalies and goal conflicts, and develop the right cognitive model towards the system. In the training include diverse sets of anomalous scenarios that show potential pitfalls and error prone problem areas. That will help the operators to learn from possible errors. Training can also help the operators for the perception of probabilistic presentation of fusion results.

\section{CONCLUSION}

Poorly designed automation can increase uncertainties in the system. These uncertainties will create additional uncertainties in the operator's mind and are likely to increase cognitive workload, reduce situation awareness, and affect human performance. The presentation of uncertain information to human operators in complex environments is a challenge. In light of theoretical, empirical and analytical studies, we gave several human factors guidelines to help operators to cope with uncertainties and help designers to develop systems that could mitigate the impact of uncertainties on human performance. Yet, more research is needed. We need to better understand the impact of uncertain information at different levels and stages of system automation. We need empirical data on the operator's reactions towards different formats of presentation of uncertain information as a function of the operator's level of expertise and culture, of the task, of the application domain (e.g., defence, medicine, business), and of the context (e.g., risk level, time pressure).

\section{REFERENCES}

Adelman, L., Bresnick, T., Black, P.K., Marvin, F.F., and Sak, S.G. 1996. Research with Patriot air defense officers: Examining information order effects. Human Factors, 38, 250-261.

Andre, A.D. and Cutler, H.A. 1998. Displaying uncertainty in advanced navigation systems. In Proceedings of the Human Factors and Ergonomics Society $42^{\text {nd }}$ Annual Meeting, Santa Monica, CA, 31-35. 
Atoyan, H., Robert J.-M., and Duquet J.-R. 2009. Comprehension of uncertainties in decision support systems. Proceeding of the IESM'2009, Montréal, Canada, 13-15 May.

Atoyan H., Duquet J.-R., and Robert J.-M. 2006. Trust in New Decision Aid Systems. In Actes de la Conférence IHM06, April 18-21, Montreal, QC, Series, ACM Press, 133, 115-122.

Atoyan, H. 2006. How to support trust in complex systems. Master's thesis, École Polytechnique de Montréal. $125 \mathrm{p}$.

Basapur, S., Bisantz, A. M., and Kesavadas, K. 2003. The Effect of Display Modality on Decision-Making in Uncertainty. Proceedings of Human Factors and Ergonomics Society.

Billings, C. E. and Woods, D. D. 1994. Concerns about adaptive automation in aviation systems. In R. Parasuraman and M. Mouloua (Eds.), Human performance in automated systems: Current research and trends. Mahwah, NJ: Erlbaum. 264-269.

Bisantz, A.S., T. Kesavadas, P. Scott, D. Lee, S. Basapur, P. Bhide, C. Sharma, and E. Roth. 2002. Holistic Battlespace Visualization: Advanced Concepts in Information Visualization and Cognitive Studies. Technical Report to the Center for Multisource Information Fusion, University of Buffalo.

Bisantz,A.M. and Kirlik,A. 1998. Investigating the Effects of Problem Format and Task Related Experience on Evidential Reasoning. In Proceedings of HICS'98, March 22-24, Dayton, Ohio.

Cosmides, L. and Tooby, J. 1996. Are humans good intuitive statisticians after all? Rethinking some conclusions from the literature on judgement under uncertainty. Cognition, 58, 1-73.

Dilla, W.N. and Stone, D.N. 1997. Representations as Decision Aids: The Asymmetric Effects of Words and Numbers on Auditors Inherent Risk Judgments Decision Sciences, 28 (3), 709-743.

Dzindolet M., Petersona, S.A., Pomranky, R.A., Pierce, L. G., and Beck, H. P. 2003. The role of trust in automation reliance. International Journal of Human Computer Studies, 58(6), 697-718.

Eick, S. and Wills, G. 1995. High Interaction Graphics, European Journal of Operations Research, 81, 445-459.

Gigerenzer, G. and Hoffrage, U. 1995. How to improve Baysian reasoning without instruction: Frequency formats. Psychological Review, 102(4), 684 - 704.

Gigerenzer, G. 1994. Why the distinction between single-event probabilities and frequences is important for psychology (and vice versa). In G. Wright \& P. Ayton (Eds.), Subjective Probabilities, 129 - 161. Chichester: John Wiley and Sons.

Gilovich, T., Griffin, D., and Kahneman, D. (Eds.). 2002. Heuristics and biases: The psychology of intuitive judgment. Cambridge, UK: Cambridge University Press.

Griethe, H. and Schumann, H. 2005. Visualizing uncertainty for improved decision making. Proceedings of the 4th International Conference on Perspectives in Business Informatics Research, University of Skovde, Sweden.

Hammond, K.R. 1993. Naturalistic Decision Making from a Brunswikian Viewpoint: Past, Present, Future. In Klein, G.A., Orasanu, J., Calderwood, R. and Zsambok, C.E. (Eds.). Decision Making in Action: Models and Methods, 205-227, Norwood, CT: Ablex. 
Holbrook, M. B. and Moore, W.L. 1981. Feature Interactions in Consumer Judgments of Verbal Versus Pictorial Presentations, Journal of Consumer Research, 8 (June), 103113.

Jarvenpaa, S.L. 1990. Graphic Displays in Decision Making: The Visual Salience Effect, Journal of Behavioral Decision Making, 3 (October-December), 247-62.

Jarvenpaa, S.L. 1989. The Effect of Task Demands and Graphical Format on Information. In Processing Strategies. Management Science. 35, No. 3 (March), 285-303.

Johnson, E.J., Meyer, R.J., and Ghose, S. 1989. When choice models fail: Compensatory representations in negatively correlated environments. Journal Marketing, 26, 255-270.

Kaber, D.B. and Endsley, M. 2004. The effects of level of automation and adaptive automation on human performance, situation awareness and workload in a dynamic control task. Theoretical Issues in Ergonomics Science, 5, 113-153.

Kahneman, D. and Tversky, A. (Eds.). 2000. Choice, values, and frames. Cambridge: Cambridge University.

Kahneman, D., Slovic, P.A., and Tversky, A. 1982. Judgment under uncertainty: Heuristics and biases. New York: Cambridge University.

Klein, G. 1998. Sources of power: How people make decisions. Cambridge, MA: MIT.

Klir, G.J. and Wierman M.J. 1999. Uncertainty-Based Information: Elements of Generalized Information Theory. Physica-Verlag/Springer-Verlag, Heidelberg and New York.

Koks, D. and Challa, S. 2005. An Introduction to Bayesian and Dempster-Shafer Data Fusion. DSTO-TR-1436, 2005, AR No. AR-012-775, November.

Lee, J.D. and See, K.A. 2004. Trust in Automation: Designing for Appropriate Reliance. Human Factors, 46, 50-80.

Lee, J. D. and Moray, N. 1992. Trust, control strategies and allocation of function in humanmachine systems. Ergonomics, 35, 1243-1270.

Liebhaber, M.J. and Feher, B. 2000. Air Threat Assessment: Research, Model, and Display Guidelines. In Proceedings of the 2000 Command and Control Research and Technology Symposium. Naval Postgraduate School, Monterey, CA.

Lind, A. T., Dershowitz, A., Chandra, D., and Bussolari, S. R. 1995. The effect of data linkprovided graphical weather images on pilot decision making. In IFAC proceedings.

Lipshitz, R. and Cohen, M.S. 2005. Warrants for prescription: analytically and empirically based approaches to improving decision making. Human Factors, 47(1), 121-130.

Lipshitz, R. and Strauss, O. 1997. Coping with Uncertainty: A Naturalistic Decision Making Analysis. Organizational Behavior and Human Decision Processing, 69, 149-163.

Lipshitz, R. 1994. Decision Making in Three Modes. Journal for the Theory of Social Behavior, 47-66.

Lurie, N.H. and Mason, C.H. 2007. Visual Representation: Implications for Decision Making. Journal of Marketing, 71 (January), 160-177.

MacEachren, A.M., Robinson, A., Hopper, S., Gardner, S., Murray, R., Gahegan, M., and Hetzler, E. 2005. Visualizing Geospatial Information Uncertainty: What We Know and What We Need to Know. Cartography and Geographic Information Science, 32, 139160 .

MacEachren, A.M. 1992. Visualizing uncertain information, Cartographic Perspective, 13, $10-19$. 
Madhavan, P. and Wiegmann, D.A. 2004. A New Look at the Dynamics of HumanAutomation Trust: Is Trust in Humans Comparable to Trust in Machines? Proceedings of the 48th Annual Meeting of the Human Factors and Ergonomics Society.

Mandel, N. and Johnson, E.J. 2002. "When Web Pages Influence Choice: Effects of Visual Primes on Experts and Novices," Journal of Consumer Research, 29 (2), 235-45.

Merritt, A. 2000. Culture in the Cockpit. Do Hofstede's Dimensions Replicate? Journal of Cross- Cultural Psychology, 31, 3, 283-301.

Miller, C. and Parasuraman, R. 2007. Designing for flexible interaction between humans and automation: Delegation interfaces for supervisory control. Human Factors, 49, 57-75.

Miller, C., Funk, H., Goldman, R., Meisner, J., and Wu, P. 2005. Implications of Adaptive vs. Adaptable UIs on Decision Making: Why "Automated Adaptiveness" is Not Always the Right Answer. In Proceedings of the 1st International Conference on Augmented Cognition, Las Vegas, NV, July, 22-27.

Moray, N., Inagaki, T., and Itoh, M. 2000. Situation adaptive automation, trust and selfconfidence in fault management of time-critical tasks. Journal of Experimental Psychology: Applied, 6(1), 44-58.

Norman, D. A. 1988. The Psychology of Everyday Things. New York: Basic Books.

Opperman, R. 1994. Adaptive user support. Hillsdale, NJ; Erlbaum.

Orasanu J. and Connolly T. 1993. The reinvention of decision making. In Gary A. Klein, Judith Orasanu, Roberta Calderwood, and Caroline E. Zsambok, editors, Decision Making in Action: Models and Methods, 3-20. Ablex Publishing Corporation.

Pang, A.T, Wittenbrink, C.M, and Lodha, K. L. 1997. Approaches to uncertainty visualization. The Visual Computer, Vol. 13, No. 8, 370-390, November, 1997.

Parasuraman, R., Sheridan, T.B. and Wickens, C.D. 2000. A model for types and levels of human interaction with automation. IEEE Transactions on Systems Man and Cybernetics-Part A: Systems and Humans, 30, 426-432.

Parasuraman, R. and Riley, V. 1997. Humans and automation: Use, misuse, disuse, abuse. Human Factors, 39, 230-253.

Payne, J.W. and Bettman, J.R. 2001. Preferential choice and adaptive strategy use. In G. Gigerenzer and R. Selten (Eds.). Bounded Rationality: The Adaptive Toolbox. MIT Press, 123-145.

Payne, J. W., Bettman, J. R., and Schkade, D. A. 1999. Measuring Constructed Preferences: Towards a Building Code. Journal of Risk and Uncertainty, 19, 243-270.

Perrin, B.M., Barnett, B.J., Walrath, L., and Grossman, J.D. 2001. Information order and outcome framing: An assessment of judgement bias in a naturalistic decision making context. Human Factors, 43, 227-238.

Riveiro, M. 2007. Evaluation of uncertainty visualization techniques for information fusion. Information Fusion, 2007 10th International Conference, 9-12 July 2007, Quebec, $1-8$.

Sager, L., Grier, R.A., Jackson, C., Levchuk, Y, and Stelzer, E. 2007. Visualization techniques for revealing uncertainty to decision makers. Proceedings of Human Systems Integration Symposium 2007, Annapolis, MD

Sheridan, T.B. and Parasuraman, 2006. R. Human-automation interaction. Reviews of Human Factors and Ergonomics, 1, 89-129. 
Sheridan, T.B. and Verplank, W.L. 1978. Human and Computer Control of Undersea Teleoperators. MIT Man-Machine Systems Laboratory, Cambridge, MA, Technical Report.

Simpson, A. 1992. HCI issues in trust and acceptability. Defence Evaluation and Research Agency, Report No. DRA TM (CAD5) 92018, November.

Stone, D. and Schkade, D. 1991. Numeric and Linguistic Information Representation in Multi-attribute Choice. Organizational Behavior and Human Decision Processes, 49, 4259.

Tegarden, D. P. 1999. Business Information Visualization, Communications of the Association for Information Systems, 1 (4).

Thomson, J., Hetzler, B., MacEachren, A., Gahegan, M. and Pavel, M. 2005. A typology for visualizing uncertainty. In Visualization and Data Analysis 2005. Proceedings of the SPIE, SPIE, IS \&T, San Jose, CA, 5669, 146-157.

Wallsten, T. S. and Budescu, D. V. 1995. A review of human linguistic probability processing: General principles and empirical evidence. The Knowledge Engineering Review, 10(1), 43 - 62.

Wallsten, T. 1990. The costs and benefits of vague information. In R. M. Hogarth (Ed.), Insights in Decision Making, 28 - 43, Chicago: University of Chicago.

Wickens, C. D. and Xu, X. 2002. Automation Trust, Reliability and Attention HMI 0203 , AHFD-02-14/MAAD-02-2, AHDF Technical Report.

Wickens, C. D., Gempler, K. and Morphew, M. E. 2000. Workload and reliability of predictor displays in aircraft traffic avoidance. Transportation Human Factors, 2, 99-126.

Yeh, M. and Wickens, C. D. 2001. Display signalling in augmented reality: Effects of cue reliability and image realism on attention allocation and trust calibration. Human Factors, 43, 355-365.

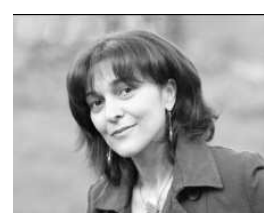

Hasmik Atoyan is a human factors consultant at Cognitive Group Inc. in Montreal, Quebec, Canada. Her work consists in applying human factors knowledge to the optimization of business processes, and to the improvement of user interfaces and the overall user experience. Mrs. Atoyan holds a Master's degree in Industrial Engineering (Cognitive Ergonomics) from École Polytechnique de Montréal, and is a $\mathrm{Ph} . \mathrm{D}$. student in the same program. She also had completed a B.A. in physics and a Master's degree in biophysics at Yerevan State University (Armenia). She worked as human factors specialist at Lockheed Martin Canada and CAE Professional Services. Her research interests are concerned with decision making in complex systems, operator's trust in new automated systems, and presentation of uncertainties in complex systems. She has several publications in journals and proceedings of international conferences and NATO workshops. 


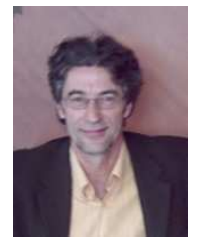

Jean-Marc Robert is full professor in Industrial Engineering at Polytechnic School of Montreal, and Director and founder of the Research Laboratory on Human-Machine Interaction at Polytechnic. He holds a B.A. and Master's degree in Psychology from the University of Montreal (Canada), a doctorate in Psychology (Cognitive Ergonomics) from University Paris V (France), and he has completed post-doctoral studies in Human Factors Engineering at NASA-Ames Research Center (California). He teaches Cognitive Ergonomics and Human-Computer Interaction to a multidisciplinary student body at Polytechnic. His research works are concerned with the themes of Accessibility, Usability, and User Experience with interactive systems in different application domains. They address questions related to user centered design, cognitive task design, collaborative work, interaction with mobile systems, and the use of virtual reality. Dr. Robert is the author of more than 170 scientific publications. He is reviewer for numerous scientific journals and conferences.

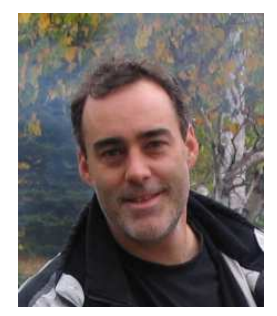

Jean-Rémi Duquet is a Staff Member at Lockheed Martin Canada, where he has worked for 15 years in the area of military Command and Control, mostly with the Research and Development department and currently as part of the Software and Systems Engineering organization. He has been involved in a variety of projects, ranging from small R\&D analyses and software technology demonstrators to large engineering projects, on which he worked in close collaboration with defense scientists and military subject matter experts. His research activities include collaborating with university researchers and co-supervising industrial graduate students in the area of Human Factors Engineering. Dr. Duquet holds a Ph.D. in Physics from Laval University (Québec, Canada), and is the author or co-author of 20 scientific publications. 